\title{
Improved double immunoenzyme labelling using alkaline phosphatase and horseradish peroxidase
}

\author{
NJ MALIK, ME DAYMON \\ From the Department of Histopathology, Royal Free Hospital, Pond Street, London NW3 2QG
}

SUMMARY Alkaline phosphatase and peroxidase complexes were applied to tissue sections simultaneously to label two antigens. An azo-coupling method was used to demonstrate the alkaline phosphatase activity. The final result gave clear contrast between the two antigens and allowed the use of haematoxylin as the nuclear counterstain.

The use of peroxidase as an enzyme label for the immunological localisation of antigens in tissue sections is a widely accepted technique. ${ }^{\prime}$ The most commonly used modification of this technique is the peroxidase antiperoxidase (PAP) method of Sternberger $e t a l,{ }^{2}$ which is believed to be the most sensitive of labelling procedures available.

Mason and Sammons ${ }^{3}$ described the use of alkaline phosphatase as an alternative enzyme label, and its use with peroxidase simultaneously to localise two antigens in the same tissue section. The disadvantages of this double labelling technique which uses Fast Blue BBN to demonstrate alkaline phosphatase activity, are the inability to apply a permanent nuclear counterstain and the necessity of mounting in an aqueous mounting medium.

In the proposed modification, both of these drawbacks have been overcome and the intensity of the alkaline phosphatase demonstration has been improved.

\section{Material and methods}

TISSUES

Thick paraffin sections $(3 \mu \mathrm{m})$ of human tonsil and lymph node, fixed in $10 \%$ phosphate-buffered neutral formalin.

BUFFERS

(a) $0.05 M$ TRIS-buffered saline (TBS), $\mathrm{pH} 7.5$, was used as the diluent for the diaminobenzidine (DAR) and for washing between incubation steps.

(b) 0.2 $M$ TRIS buffer, pH 9.0, was used in the demonstration of alkaline phosphatase activity.

ANTISERA

Sera were obtained from several commercial

Accepted for publication 28 January 1982 sources: Rabbit antisera to various human antigens; swine antirabbit immunoglobulins; rabbit PAP complex; normal swine serum (Dakopatts $\mathrm{A} / \mathrm{s}$ ).

Goat antisera to various human antigens; donkey antigoat IgG (Miles Laboratories Ltd).

Goat antialkaline phosphatase (Northeast Biomedical Laboratories Ltd).

ENZYMES

Alkaline phosphatase $(3.0 \mathrm{U} / \mathrm{mg}$ activity) from calf intestine (Miles Laboratories Ltd).

Trypsin, type II pancreatic (Sigma London Chemical Co Ltd).

\section{Substrates and capture reagents}

Naphthol AS-TR phosphoric acid, sodium salt (Sigma London Chemical Co Ltd).

New Fuchsin (CI 42520) (Raymond A Lamb).

$3,4, \quad 3$ ',4'-tetra-aminobiphenyl hydrochloride

(DAB) (British Drug Houses Chemicals Ltd).

\section{METHOD}

The technique employed was a standard PAP reaction, ${ }^{2}$ in conjunction with a similar method using an alkaline phosphatase antialkaline phosphatase complex as the enzyme label. ${ }^{3}$ Many pairs of antigens can be demonstrated by varying the first layer antisera only. This is illustrated in the example procedure below, outlining the demonstration of kappa and lambda light chains.

\section{Procedure}

1 Dewax sections and block endogenous peroxidase with $3 \% \mathrm{H}_{2} \mathrm{O}_{2}$ in methanol.

2 Trypsinise with $0.1 \%$ trypsin in $0.1 \%$ calcium chloride, $\mathrm{pH} 8.6$, at $37^{\circ} \mathrm{C}$.

3 Apply normal swine serum, diluted $1 / 10$ in TBS.

4 Apply goat antihuman kappa light chains and rabbit antihuman lambda light chains. 
5 Wash and apply donkey antigoat IgG and swine antirabbit immunoglobulins.

6 Wash and apply rabbit PAP complex and goat antialkaline phosphatase diluted in $5 \mathrm{mg} / \mathrm{ml}$ alkaline phosphatase.

7 Wash and demonstrate alkaline phosphatase activity.

8 Wash and demonstrate peroxidase activity.

9 Wash, counterstain with haematoxylin, dehydrate, clear in xylene and mount.
Kappa light chains
red
Lambda light chains brown
Nuclei
blue

Incubation in stages 4,5 , and 6 (30 min each), consisted of the two different antisera mixed together, in TBS, to give the working dilution for both.

-for example, goat antihuman kappa light chains, $1 / 500$; rabbit antihuman lambda light chains, $1 / 1000$.

This would be composed of:

TRIS-buffered saline $1 \mathrm{ml}$.

Goat antihuman kappa light chains $2 \mu \mathrm{l}$.

Rabbit antihuman lambda light chains $1 \mu$ l.

In stage 6, the alkaline phosphatase goat antialkaline phosphatase complex was prepared $60 \mathrm{~min}$ before use and the rabbit PAP complex added just prior to application.

The alkaline phosphatase activity was demonstrated using a simultaneous capture method, (see below) modified from Stutte 4 (G Reynolds, personal communication 1980). It utilised a substituted naphthol as the substrate and a diazonium salt as the capture reagent.

\section{Simultaneous capture method \\ Incubating medium \\ $4 \%$ New Fuchsin in $2 \mathrm{M} \mathrm{HCl}$ \\ $4 \%$ sodium nitrite \\ $250 \mu \mathrm{l}$ \\ Shake to allow hexanitrogenation. \\ $0 \cdot 2 M$ TRIS $\mathrm{HCl}$ buffer, $\mathrm{pH} 9 \cdot 0$ \\ naphthol AS-TR phosphoric acid \\ dissolved in $0.2 \mathrm{ml}$ dimethylformamide. \\ $250 \mu \mathrm{l}$ \\ $40 \mathrm{ml}$ \\ $10 \mathrm{mg}$}

Sections were immersed in the filtered, incubating medium for $10 \mathrm{~min}$ at room temperature, followed by thorough washing with distilled water.

Peroxidase activity was demonstrated using DAB $(0.2 \mathrm{mg} / \mathrm{ml}) . \mathrm{H}_{2} \mathrm{O}_{2}$ was used as the substrate at a concentration of $0.01 \%$.

\section{Results}

Using the modification described, alkaline phosphatase labelling was seen as a deep red colour, which contrasted well with the brown reaction product of the peroxidase activity. Both of these col- oured products were resistant to decolourisation in water, alcohol, acid-alcohol and xylene; therefore haematoxylin counterstaining was possible and resinous mounting media could be used. The end result was an attractive, strongly stained section, showing clear nuclear detail and not prone to either fading in intensity or-diffusion of the coloured products.

When the peroxidase activity was demonstrated first, the subsequent alkaline phosphatase activity appeared to be diminished. When the order of the enzyme reactions was reversed, no reduction in the peroxidase activity was observed.

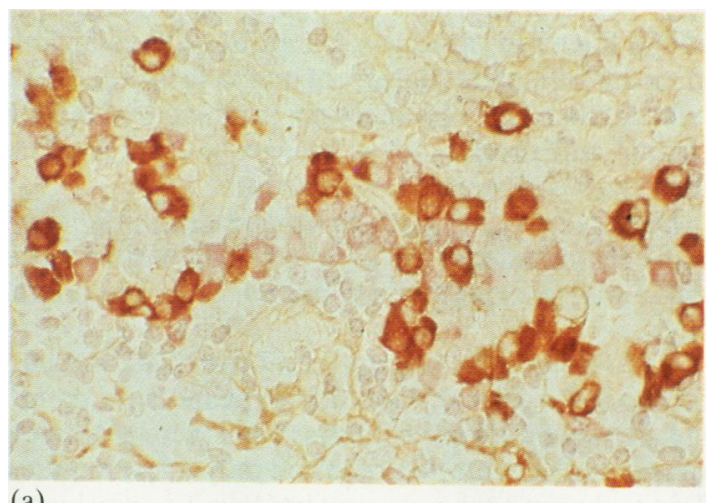

(a)

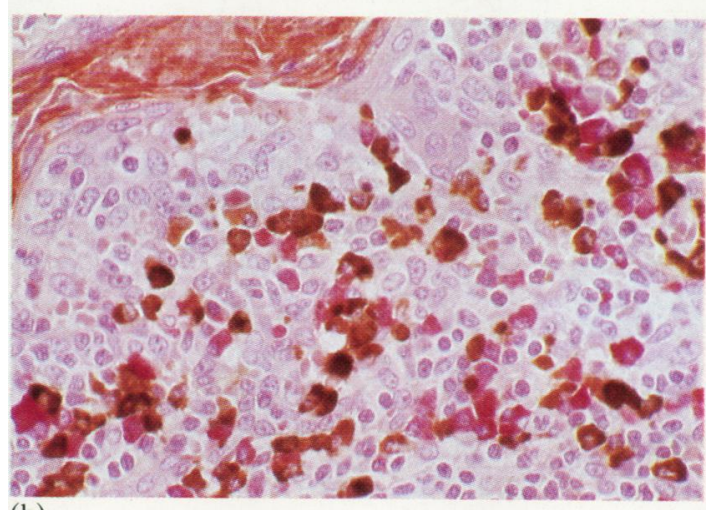

(b)

Tonsil; kappa light chains labelled with alkaline phosphatase, New Fuchsin (red). Lambda light chains labelled with peroxidase, Diaminobenzidine (brown). (a) Peroxidase demonstration followed by alkaline phosphatase demonstration. Original magnification $\times 160$.

(b) Alkaline phosphatase demonstration followed by peroxidase demonstration. Original magnification $\times 160$.

\section{Discussion}

Using the technique of Mason and Sammons, it was found that the demonstration of peroxidase activity inhibited the subsequent demonstration of peroxidase activity (Fig. a). However, by reversing the 
order in which the two reactions were performed no interference appeared to occur (Fig. b). The most likely cause of this effect is $\mathrm{H}_{2} \mathrm{O}_{2}$, which is used as the substrate for peroxidase; $\mathrm{H}_{2} \mathrm{O}_{2}$ acts as a poison to most enzymes and if exposed to the alkaline phosphatase, rather than its reaction product, it impairs the enzyme's activity and hence a weaker final result is obtained.

By demonstrating alkaline phosphatase activity first, the reactions of both Fast Blue BBN and New Fuchsin as capture reagents were greatly enhanced. Employing New Fuchsin, rather than Fast Blue $\mathrm{BBN}$, alkaline phosphatase as an enzyme label becomes as useful and reliable as peroxidase. The ability to employ a nuclear counterstain is invaluable in determining the nature of the cell labelled. We believe that the red reaction product obtained is clearer and produces less background interference than the DAB peroxidase reaction.

Despite using a methanol/peroxide block, some red cell peroxidase activity was still visible. This effect was so slight that it could not cause any confusion.

At first sight the lengthy list of materials and buffers necessary for this method appears quite daunting. However, all the solutions used were extremely simple and preparation required only a few minutes. The technique takes approximately $15 \mathrm{~min}$ longer than a single immunoenzyme method as the only extra stage is the demonstration of the second label.

Our experimentation has so far been solely confined to immunoglobulins, as their presence can be almost certainly assured in normal, control material. However some work has already been carried out on practical applications of double immunoenzymatic staining techniques. ${ }^{5}$

To confirm our findings, single immunoperoxidase labelling was carried out on two consecutive sections of the control material. When the two immunoglobulins demonstrated in the double labelling technique were demonstrated singly, excellent corroboration was obtained.

We would like to express our gratitude to Miss Jackie Lewin and $\mathrm{Mr}$ Paul Bates for their photographic assistance. We are also extremely grateful to Mercia Brocades Ltd, Northeast Biomedical Laboratories Ltd, and Miles Laboratories Ltd for the generous contributions towards the cost of the colour plates.

\section{References}

'Heyderman E. Immunoperoxidase technique in histopathology: applications, methods, and controls. J Clin Pathol 1979;32:971-8.

${ }^{2}$ Sternberger LA, et al. The unlabelled antibody method of immunocytochemistry. J Histochem Cytochem 1970;18:31533.

${ }^{3}$ Mason DY, Sammons R. Alkaline phosphatase and peroxidase for double immunoenzymatic labelling of cellular constituents. $J$ Clin Pathol 1978;31:454-60.

4 Stutte HJ. Hexatotiertes triamino-tritoylmethanchlorid(neufuchsin) als Kupplungsalz in der Fermenthistochemiece Histochemie 1967;8:327-31.

${ }^{5}$ Mason DY, et al An immunohistological study of human lymphoma. Clin Exp Immunol 1980;40:235-48.

Requests for reprints to: Mr M Daymon, c/o Department of Histopathology, The Royal Free Hospital, Pond Street, London NW3 2QG, England. 